\title{
CORRELAÇÃO ENTRE OS ÍNDICES DE NECROSE E A ESTABILIZAÇÃO PRECOCE NAS FRATURAS DA EXTREMIDADE PROXIMAL DO FÊMUR NA INFÂNCIA
}

\author{
CORRELATION BETWEEN AVASCULAR NECROSIS AND EARLY STABILIZATION \\ OF PROXIMAL FEMORAL FRACTURES IN CHILDHOOD
}

Diego da Costa Astur', Gustavo Gonçalves Arliani', Carolina Lins e Silva Nascimento², Francesco Camara Blumetti ${ }^{3}$, Marcio José Alher Fonseca ${ }^{3}$, Eiffel Tsuyoshi Dobashi ${ }^{4}$, José Antonio Pinto ${ }^{5}$, Akira Ishida ${ }^{6}$

\section{RESUMO}

Objetivo: Desenvolvemos este trabalho, com o intuito de avaliar o resultado do tratamento de pacientes portadores de fraturas do fêmur proximal, em uma série de casos. Procuramos observar a influência das complicações mais prevalentes nos resultados finais após o mínimo de dois anos de seguimento. Correlacionamos especialmente a instalação da necrose avascular e o tempo entre o acidente e a instituição da terapêutica. Métodos: Estudamos, retrospectivamente, 29 pacientes com fraturas da extremidade proximal do fêmur, com idade inferior a 14 anos entre 1988 e 2007. Analisamos as seguintes variáveis: sexo, idade, mecanismo de trauma, classificação da fratura (Delbet), tratamento realizado, complicações (pseudartrose, deformidade em varo, anisomelia e necrose avascular), tempo para cirurgia e resultado (Ratliff). Obtivemos uma análise descritiva individual de cada variável. Os testes foram utilizados de acordo com a adequação das premissas de normalidade e para avaliação utilizamos o teste exato de Fisher. Resultados: Obtivemos cinco (17,2\%) pacientes com necrose avascular sendo três $(60,0 \%)$ com idade superior a 10 anos; $73,3 \%$ dos pacientes tratados nas primeiras 24 horas apresentaram bons resultados; a causa mais comum de fratura foi acidente automobilístico (44,8\%); os melhores resultados foram observados nos pacientes tratados cirurgicamente; 41,4\% evoluíram com algum tipo de complicação. Conclusões: Entre os 29 pacientes tratados, segundo os critérios de Ratliff,

\section{ABSTRACT}

Objective: This study was developed with the main purpose of evaluating treatment results of proximal femoral fractures in a series of cases. We sought to observe the influence of the most frequent complications on the final results after a minimum follow-up of 2 years. We have especially considered the relationship between development of avascular necrosis and time between the accident and therapeutic intervention. Method: We retrospectively studied proximal femoral fractures in 29 patients under 14 years of age from 1988 to 2007. The following parameters were analyzed: sex, age, mechanism of injury, fracture classification (Delbet), treatment, complications (pseudoarthrosis, coxa vara, leg length discrepancy and avascular necrosis), time for surgery, and results (Ratliff). Statistical analysis was performed according to the descriptive evaluation of each parameter by using Fisher's exact test. Results: Five (17.2\%) patients had avascular necrosis, 3 of whom (60.0\%) were older than 10 years of age. Seventy-three point three percent of patients treated in the first 24 hours showed good results. The most common cause of fracture was traffic accident (44.8\%). The best results were observed in patients who were treated surgically; $41.4 \%$ developed some type of complication. Conclusions: Among the 29 patients treated, 58.6\% had good, $27.6 \%$ had regular and $13.8 \%$ had poor results according

\footnotetext{
1 - Médico Residente do Departamento de Ortopedia e Traumatologia da Universidade Federal de São Paulo - Escola Paulista de Medicina, São Paulo, SP.

2 - Aluna do Curso de Graduação em Medicina da Universidade Federal de São Paulo - Escola Paulista de Medicina, São Paulo, SP.

3 - Médico Ortopedista do Departamento de Ortopedia e Traumatologia da Universidade Federal de São Paulo - Escola Paulista de Medicina, São Paulo, SP.

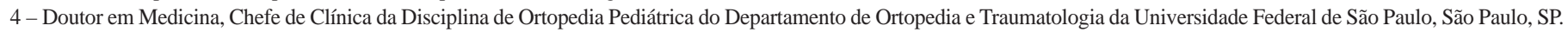

5 - Professor Adjunto, Chefe da Disciplina de Ortopedia Pediátrica do Departamento de Ortopedia e Traumatologia da Universidade Federal de São Paulo, São Paulo, SP.

6 - Professor Titular e Chefe do Departamento de Ortopedia e Traumatologia da Universidade Federal de São Paulo, São Paulo, SP.
} 
obtivemos 58,6\% de bons, $27,6 \%$ de regulares e $13,8 \%$ de maus resultados. Quando aplicado o tratamento incruento, obtivemos apenas $17,0 \%$ de bons resultados, enquanto que após o tratamento cirúrgico obtivemos 69,3\%. Da mesma forma, observamos que houve $73,3 \%$ de bons resultados quando a cirurgia foi realizada nas primeiras 24 horas e apenas $42,8 \%$ nos pacientes submetidos à intervenção terapêutica após este período. Pacientes submetidos à cirurgia nas primeiras 24 horas evoluíram com necrose da cabeça do fêmur em 13,3\%, enquanto os que foram operados após este período tiveram esta complicação em 21,4\% dos casos.

Descritores - Fraturas do fêmur/epidemiologia; Fraturas do fêmur/cirurgia; Criança to Ratliff criteria. When conservative treatment was applied, only $17.0 \%$ had good results, while surgical intervention results were $69.3 \%$ good. In addition, we obtained $73.3 \%$ good results when surgery was performed within the first 24 hours and only $42.8 \%$ good results in patients submitted to surgery after this period. Patients operated in the first 24 hours developed avascular necrosis in 13.3\% of cases, while $21.4 \%$ of those operated after that period developed this complication.

Keywords - Femoral fractures/epidemiology; Femoral fractures/surgery; Child

\section{INTRODUÇÃO}

As fraturas do fêmur proximal na criança são graves e estão associadas com altos índices de complicações. Geralmente são ocasionadas por traumas que envolvem alta energia cinética como quedas de altura, acidentes automobilísticos e a prática de esportes radicais, podendo acarretar, também, importantes lesões dos tecidos moles (nervos, artérias, vísceras e trato geniturinário) ${ }^{(1-4)}$. Felizmente, estas lesões não são frequentes na infância e representam menos de $1 \%$ de todas as fraturas que afetam o esqueleto imaturo ${ }^{(5)}$.

Desde o início do tratamento devemos agir de forma a prevenir complicações imediatas e, principalmente, as tardias como: pseudoartrose, coxa vara, consolidação viciosa e o fechamento fisário prematuro ${ }^{(6-8)}$.

Destas, a necrose avascular é a única que, do momento que iniciou seu curso, não pode ser evitada, mesmo quando é efetuado um atendimento rápido e adequado. Destacamos que esta condição, dependendo da sua extensão e topografia, determina resultado futuro desfavorável. Considerando que o fêmur proximal, nesta faixa etária, encontra-se em franco desenvolvimento, as deformidades decorrentes destas complicações podem agravar-se com a progressão da idade e do crescimento.

A correlação entre o surgimento da necrose avascular do fêmur proximal com o tempo decorrido até a estabilização destas fraturas, não é clara na literatura. Alguns autores consideram que seu desenvolvimento já seria estabelecido no momento do acidente, e o desvio inicial entre os fragmentos fraturados fosse determinante para a ocorrência de complicações, devido ao dano circulatório nesta região. Quando a estabilização cirúrgica é necessária nas fraturas do colo femoral em indivíduos adultos, sabe-se que a precocidade na instituição da terapêutica é determinante para minimizar os índices de necrose avascular. Entretanto, esta premissa ainda não foi amplamente estudada em crianças.

Desenvolvemos este trabalho com o intuito de avaliar, numa série de casos, o resultado do tratamento de pacientes portadores de fraturas da extremidade proximal do fêmur. Procuramos observar a influência das complicações mais prevalentes no resultado, após o período mínimo de dois anos de seguimento. Correlacionamos, especialmente, o estabelecimento da necrose avascular e o tempo entre o acidente e a instituição da terapêutica.

\section{MATERIAL E MÉTODOS}

Inicialmente, o projeto desta pesquisa foi submetido à avaliação e aprovado para realização, pelo Comitê de Ética em Pesquisa de nossa Instituição.

Trata-se de um estudo retrospectivo, no qual apresentamos 29 pacientes com fraturas da extremidade proximal do fêmur e idade inferior a 14 anos, tratados entre os anos de 1988 e 2007. Do total, 18 (62,1\%) são do sexo masculino e 11 (37,9\%) do feminino, cuja média das idades é 8,68 anos, variando de seis meses até 14 anos (Tabela 1).

$\mathrm{O}$ acidente automobilístico correspondeu à principal causa de lesão, envolvendo 13 pacientes (44,8 \%); seguida da queda de altura, com 11 pacientes $(37,9 \%)$; acidentes com bicicleta em três pacientes (10,3\%); e crise convulsiva em dois pacientes (6,9\%).

Para a caracterização destas lesões, utilizamos a classificação de Delbet apud Colonna ${ }^{(9)}$ e sua distribuição encontra-se ilustrada na Figura 1. 
Tabela 1 - Os 29 pacientes e as características da fratura estudadas

\begin{tabular}{|c|c|c|c|c|c|c|c|c|c|c|c|c|}
\hline Nome & Sexo & Idade & $\begin{array}{l}\text { Mecanismo } \\
\text { trauma }\end{array}$ & Classificação & Tratamento & $\begin{array}{l}\text { Método } \\
\text { Fixação }\end{array}$ & $\begin{array}{l}\text { Coxa } \\
\text { Vara }\end{array}$ & ANISO & NACF & Follow up & Cirurgia & Resultado \\
\hline MAJ & $\mathrm{F}$ & $5+6$ & Queda de altura & II & RAFI & $\mathrm{PC}+\mathrm{FK}$ & & & & 5 & + & B \\
\hline CFA & M & 10 & Atropelamento & II & GPP & & + & & & 3 & - & $\mathrm{R}$ \\
\hline CJG & M & 12 & Atropelamento & II & RAFI & PC & & & & 4 & + & $B$ \\
\hline $\mathrm{CSC}$ & $\mathrm{F}$ & 14 & Atropelamento & II & GPP & & & & I & 2 & + & M \\
\hline MMS & $\mathrm{F}$ & 7 & Queda de altura & II & RAFI & PC & & & & $7+6$ & + & $B$ \\
\hline ECM & $\mathrm{F}$ & $11+4$ & Atropelamento & II & RAFI & PC & & & & 5 & + & $B$ \\
\hline RJG & M & $6+2$ & Atropelamento & II & RAFI & PC & + & + & & $3+6$ & + & $\mathrm{R}$ \\
\hline WPD & $M$ & $7+6$ & Atropelamento & II & RAFI & PC & & & & $3+6$ & + & M \\
\hline FSB & M & $6+9$ & Ac. ciclístico & II & RAFI & PC & & & & 4 & + & B \\
\hline RLO & $\mathrm{F}$ & $7+6$ & Atropelamento & III & GPP & PC & + & & & 3 & - & $\mathrm{R}$ \\
\hline DAS & M & 7 & Queda de altura & II & RAFI & PC & & & & 8 & + & B \\
\hline SS & M & 14 & Crise convulsiva & IV & RAFI & $\mathrm{PC}+\mathrm{FK}$ & & & & $7+6$ & - & $B$ \\
\hline GTS & $\mathrm{F}$ & $0+6$ & Crise convulsiva & 1 & GPP & & + & & + & 2 & - & $M$ \\
\hline IMC & $\mathrm{F}$ & $5+9$ & Queda de altura & II & GPP & & & & & $3+6$ & + & $B$ \\
\hline $\mathrm{IR}$ & $M$ & $12+11$ & Queda de altura & II & RAFI & PC & & & + & $3+6$ & - & $\mathrm{R}$ \\
\hline WLS & M & 12 & Atropelamento & II & RAFI & PC & & & & 3 & - & $B$ \\
\hline RRP & M & 8 & Queda de altura & II & RAFI & PC & & & & 2 & - & B \\
\hline MAS & $\mathrm{F}$ & 5 & Queda de altura & II & RAFI & PC & & & & 2 & + & $B$ \\
\hline JCS & M & 7 & Queda de altura & III & RAFI & PC & + & + & & $2+1$ & - & $R$ \\
\hline MMS & M & 5 & Queda de altura & III & RAFI & PC & & & & 2 & + & B \\
\hline THBS & M & 14 & Atropelamento & III & RAFI & PC & & & & 3 & - & B \\
\hline CFS & M & 14 & Ac. ciclístico & II & RAFI & $P C$ & & & & 2 & + & $B$ \\
\hline AAR & $\mathrm{F}$ & 14 & Atropelamento & I & RAFI & $\mathrm{FK}$ & & & + & 2 & + & $\mathrm{R}$ \\
\hline DS & M & 5 & Queda de altura & IV & RAFI & PC & + & + & & 4 & - & B \\
\hline VBSO & M & 6 & Queda de altura & III & RAFI & PC & & & & 5 & + & $B$ \\
\hline sos & $\mathrm{F}$ & 12 & Atropelamento & IV & RAFI & $\begin{array}{c}\text { Placa + } \\
\text { parafuso }\end{array}$ & & & & 5 & - & $\mathrm{R}$ \\
\hline RS & M & 7 & Atropelamento & II & RAFI & PC & + & + & & 2 & - & $B$ \\
\hline LRFS & $\mathrm{F}$ & 8 & Ac. Ciclístico & II & RAFI & PC & & + & + & 2 & - & M \\
\hline JSC & $M$ & 7 & Atropelamento & II & GPP & PC & + & + & & $6+2$ & - & $\mathrm{R}$ \\
\hline
\end{tabular}

Fonte: Dados coletados dos 29 pacientes com fratura da extremidade proximal do fêmur.

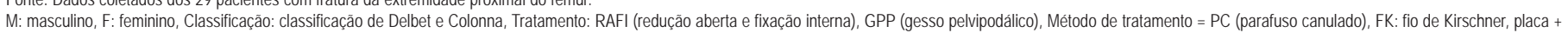

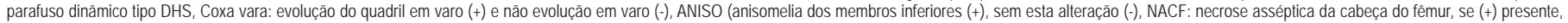
se (-) ausente, follow up: tempo de seguimento, Cirurgia +: cirurgia nas primeiras 24 horas, Cirurgia -: cirurgia após 24 horas), resultado: B - bom, R - regular, M - ruim.

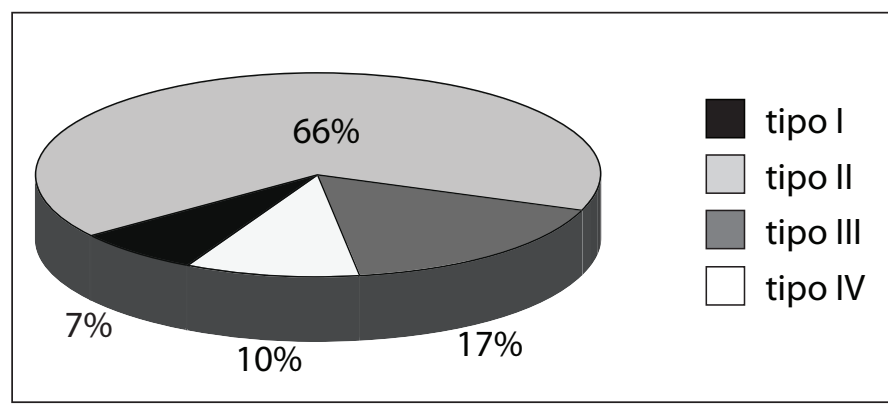

Classificação de Delbet e Colonna - Distribuição dos pacientes

Figura 1 - Distribuição dos padrões de fratura de acordo com a classificação de Delbet e Colonna

Todos os pacientes foram submetidos ao tratamento de suas fraturas no centro cirúrgico, sob anestesia geral, de acordo com a idade e a característica da fratura. O tratamento incruento foi realizado pela confecção de aparelho gessado pelvipodálico, precedido pela redução fechada da fratura quando necessária. A estabilização cirúrgica foi obtida pela fixação interna com parafusos esponjosos, canulados ou parafuso deslizante (Figura 2). Todos foram mantidos imobilizados com gesso pelvipodálico, entre seis e oito semanas, período em que foi observada a consolidação das fraturas.

O período mínimo de seguimento ambulatorial foi de dois anos e o máximo de oito anos com média de três anos e meio. A avaliação dos resultados foi baseada na classificação descritiva proposta por Ratliff( ${ }^{(10)}$ :

- Bom: pacientes sem dor, mínima restrição de 
movimento do quadril, atividades normais do dia a dia e imagem radiológica normal, ou com leve deformidade do colo do fêmur.

- Regular: pacientes com dor ocasional, restrição importante do quadril, mas com arco de movimento maior que $50 \%$, atividades normais do dia a dia, e deformidade importante do colo do fêmur com sinais leves de necrose da cabeça femoral.

- Mau: pacientes com dor debilitante, menos que 50\% de arco de movimento do quadril, atividades restritas e necrose severa da cabeça do fêmur com artrose importante do quadril.

Para a análise descritiva foram calculados: média, desvio padrão, mediana para idade e tempo de seguimento. Pela aplicação do teste exato de Fisher procuramos estabelecer correlações entre os resultados obtidos e os seguintes parâmetros: classificação, tipo de tratamento e tempo para o tratamento (até 24 horas e > 24 horas).
Da mesma maneira, aplicamos o teste exato de Fisher, buscando estabelecer correlações entre a presença de necrose avascular da cabeça do fêmur com os seguintes parâmetros: classificação, tipo de tratamento e tempo para o tratamento (até 24 horas e menor que 24 horas).

\section{RESULTADOS}

Em nossa casuística houve predomínio do tratamento cirúrgico (79,3\%) com relação ao tratamento não cirúrgico (20,7\%). Quanto ao tempo para o tratamento, 15 pacientes $(51,7 \%)$ foram submetidos ao procedimento cirúrgico nas primeiras 24 horas.

Entre os 29 pacientes tratados, segundo os critérios de Ratliff ${ }^{(10)}$, obtivemos 58,6\% bons, 27,6\% regulares e 13,8\% maus resultados (Tabela 2).

Considerando o tratamento incruento obtivemos $17,0 \%$ bons, $50,0 \%$ regulares e $33,0 \%$ maus resultados.
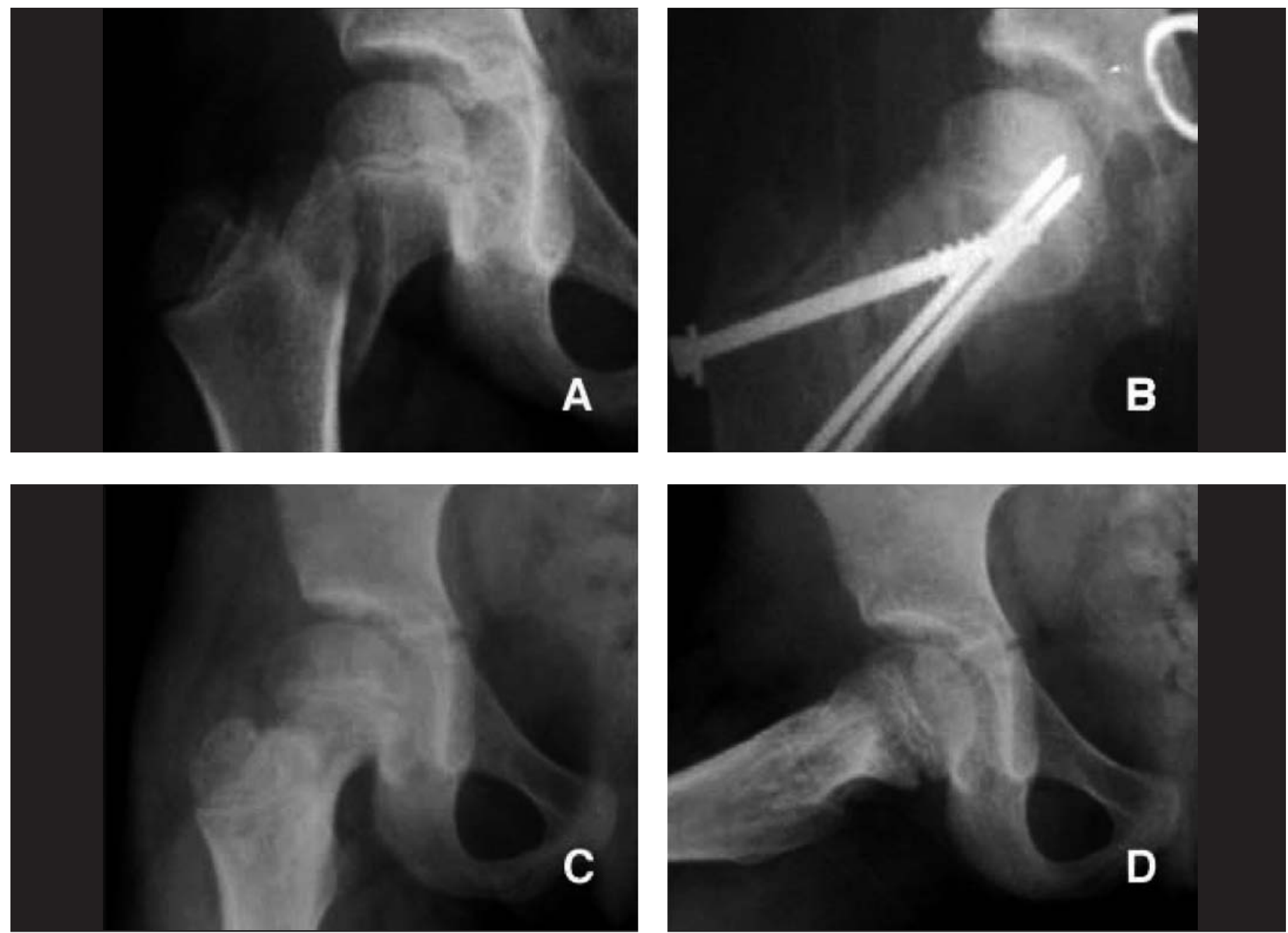

Figura 2 - Paciente MAJ, sexo feminino, com cinco anos e seis meses, apresentando fratura do fêmur proximal direito. (A) Aspecto pré-operatório. (B) Aspecto pós-operatório imediato. (C e D) Aspecto pós-operatório tardio com cinco anos de evolução, após a retirada do material de síntese. 
Entre aqueles tratados cirurgicamente obtivemos 69,3\% bons, $21,0 \%$ regulares e $8,7 \%$ maus resultados.

Observamos que 12 (41,4\%) dos nossos pacientes evoluíram com complicações, sendo oito $(27,6 \%)$ pacientes com coxa vara, seis (20,7\%) com anisomelia, cinco $(17,2 \%)$ com necrose avascular do fêmur proximal, sendo uma $(3,4 \%)$ do tipo IA de Delbet apud Colonna ${ }^{(9)}$, duas $(6,9 \%)$ do tipo II e duas $(6,9 \%)$ do tipo III.

Com relação ao tempo de cirurgia, tivemos 73,3\% de bons resultados, quando a cirurgia foi realizada nas primeiras 24 horas. Para aqueles que foram submetidos à terapêutica operatória, apenas após este período, 42,8 $\%$ apresentaram bons resultados.

Foi realizado um estudo comparativo entre os tipos de fratura, de acordo com a classificação de Delbet apud Colonna $^{(9)}$, com a evolução ou não da cabeça do fêmur para necrose (Tabela 3).

Com relação ao tratamento, o índice de necrose entre os pacientes tratados apenas com gesso pelvipodálico foi de 34,0\%. Para aqueles submetidos à redução aberta e fixação interna, tivemos $13 \%$ dos casos evoluindo com necrose.

Pacientes submetidos à cirurgia nas primeiras 24 horas evoluíram com necrose da cabeça do fêmur em $13,3 \%$, enquanto os que foram operados após este período tiveram esta complicação em $21,4 \%$ dos casos.

Tabela 2 - Classificação de Delbet e Colonna correlacionada com os resultados baseados nos parâmetros de Ratliff

\begin{tabular}{c|c|c|c|c}
\hline $\begin{array}{c}\text { Classificação } \\
\text { x resultado }\end{array}$ & Bom & Regular & Ruim & Total \\
\hline I & 0 & $1(50 \%)$ & $1(50 \%)$ & 2 \\
\hline II & $12(63,5 \%)$ & $4(21 \%)$ & $3(15,7 \%)$ & 19 \\
\hline III & $3(60 \%)$ & $2(40 \%)$ & 0 & 5 \\
\hline IV & $2(66,7 \%)$ & $1(33,3 \%)$ & 0 & 3 \\
\hline Total & $17(58,6 \%)$ & $4(13,7 \%)$ & $8(27,7 \%)$ & 29 \\
\hline
\end{tabular}

Tabela 3 - Distribuição das fraturas de acordo com a classificação de Delbet e Colonna correlacionada com presença ou ausência de necrose asséptica da cabeça do fêmur

\begin{tabular}{c|c|c|c}
\hline Tipo & Não & Sim & Total \\
\hline I & 0 & $2(100 \%)$ & 2 \\
\hline II & $16(84,2 \%)$ & $3(15,8 \%)$ & 19 \\
\hline III & $5(100 \%)$ & 0 & 5 \\
\hline IV & $3(100 \%)$ & 0 & 3 \\
\hline Total & $24(82,8 \%)$ & $5(17,2 \%)$ & 29 \\
\hline
\end{tabular}

Não observamos necrose da cabeça do fêmur entre os pacientes que obtiveram bons resultados. Encontramos três casos de necrose $(75,0 \%)$ entre os que obtiveram maus resultado. Entre os resultados considerados regulares, dois (25\%) evoluíram com necrose.

\section{DISCUSSÃO}

Segundo dados da fundação Seade, o atropelamento é a terceira principal causa de óbito em crianças no estado de São Paulo. Estes números vêm decaindo na última década, embora ainda representem potencial fonte de trauma de alta energia, nas faixas etárias entre cinco-nove anos e 10-14 anos. Em nossa série de casos encontramos, predominantemente, pacientes vítimas de atropelamento e queda de altura, o que coincide com o relatado na literatura ${ }^{(11-13)}$. Entretanto, observamos em nosso estudo quatro pacientes cujas lesões ocorreram por traumas de menor energia (queda de bicicleta e crises convulsivas), o que não é habitual.

Constatamos que os piores resultados foram observados nos dois pacientes com lesões transfisárias (tipo I). Nos portadores de fraturas dos tipos II, III e IV notamos bons resultados em 2/3 dos casos. Entretanto, ressaltamos que pelo número restrito de fraturas do tipo I, não alcançamos resultado estatisticamente significante, quando utilizado o teste exato de Fisher $(p=0,446)$.

As fraturas do colo femoral cursam frequentemente com o dano circulatório para a cabeça femoral, devido ao comprometimento do suprimento sanguíneo, intra-ósseo e extra-ósseo. Embora 11 a 40\% das fraturas sejam acompanhadas pela ruptura capsular, a maioria cursa com hemartrose que proporcionaria uma elevação da pressão intracapsular. Este fenômeno poderia aumentar, segundo alguns autores, o risco de necrose avascular.

Não realizamos rotineiramente artrocentese em busca da minimização da pressão intracapsular. Não há indícios relevantes na literatura ortopédica, que suporte este procedimento, como elemento que reduza os índices de necrose. Acredita-se que a ruptura capsular estaria associada aos traumas de alta energia, como postulado por Crawford et al ${ }^{(14)}$, que encontraram maior fluxo sanguíneo para a cabeça femoral nas fraturas classificadas como Garden I e II em idosos. 
De fato, as fraturas estágios Garden III e IV em adultos, apresentam um índice de necrose de $75 \%$ e 100\%, respectivamente, quando o tratamento é instituído após 24 horas do acidente.

Os melhores resultados nesta série, também foram observados naqueles tratados de forma cirúrgica. Estes apresentaram evolução satisfatória em 69,3\% dos casos, sendo essa correlação estatisticamente significante quando aplicado o teste exato de Fisher ( $p=0,045$ ). Embora 64,71\% dos pacientes, com boa evolução, tenham sido observados no grupo operado precocemente, este dado não apresentou significância estatística $(\mathrm{p}=0,183)$.

Procuramos avaliar em nossa pesquisa, os elementos potencialmente associados com o aparecimento da necrose avascular. Não foi possível estabelecer uma correlação entre a ocorrência desta afecção com o método terapêutico utilizado $(\mathrm{p}=0,651)$.

Desde 1936, Mitchell ${ }^{(15)}$ ressalta a importância do tratamento precoce das fraturas do fêmur proximal, visando melhor prognóstico. Nosso estudo procurou correlacionar a possível redução das taxas de necrose avascular com a estabilização das fraturas em até 24 horas, como presumia este autor. Entretanto, não observamos correlação entre a ocorrência desta complicação com o tempo decorrido entre a fratura e o tratamento ( $p=0,269)$. Estes resultados coincidem com o relato de outros autores ${ }^{(16)}$, que também não observaram esta associação, sendo relatado como únicos fatores preditivos para o desenvolvimento da necrose, a idade do paciente e o tipo de fratura $26^{(10,13,17)}$.

Porém, ao analisarmos, individualmente, os pacientes tratados cirurgicamente que evoluíram com resultado desfavorável e necrose avascular (pacientes número 13 e 28), percebemos que ao aplicarmos a classificação prognóstica de Boitzy ${ }^{(18)}$, o desvio entre os fragmentos fraturados nas radiografias iniciais mantinham um contato inferior a 50\%. Esta circunstância determina que uma alta energia cinética dissipou-se pela região proximal do fêmur, ocasionando um dano circulatório, já no momento dos acidentes.

Apenas cinco de nossos pacientes (17,2\%) desenvolveram necrose do fêmur proximal, sendo que três (60,0\%) apresentaram resultados regulares e apenas dois (40,0\%) apresentaram evolução desfavorável.
Entretanto, quando correlacionados nossos achados funcionais com a presença de necrose, observamos uma associação estatisticamente significante entre as duas variáveis, pois, observamos maior número de bons resultados (70,83\%) no grupo que não desenvolveu esta complicação ( $p=0,002)$.

O estudo de Ratliff ${ }^{(10)}$ afirma que não há como prever ou evitar o desenvolvimento da necrose avascular, observando esta complicação em 42,0\%(6). Contudo, mais recentemente, a necrose tem sido relatada em menor número. Os fatores mais comumente implicados com o surgimento desta complicação têm sido: tipo de fratura, deslocamento inicial, tratamento empregado, idade do paciente e tempo decorrido até o tratamento $^{(13,16,19)}$.

Observamos em nossa amostra, que a maioria dos casos de necrose $(60,0 \%)$ ocorreu em pacientes acima dos 10 anos de idade, o que coincide com o relatado por outros estudos ${ }^{(10,13,17)}$. Entretanto, não houve significância estatística para este achado. Por outro lado, obtivemos uma associação positiva entre o tipo de fratura e a ocorrência de necrose, visto que esta foi mais comum nas fraturas do tipo I de Delbet apud Colonna $^{(9)}(p=0,047)$. No estudo de Moon ${ }^{(16)}$, o autor estabeleceu que o risco de necrose para fraturas do tipo I, II, e III, eram respectivamente 15 , seis e quatro vezes maior do que as fraturas do tipo IV.

Por serem lesões pouco comuns, as publicações sobre este tema limitam-se às avaliações retrospectivas de séries com poucos casos. Nas últimas décadas constatamos uma série de inovações na cirurgia ortopédica, como a introdução de técnicas menos traumáticas, utilização de intensificador de imagem, melhora da agilidade dos serviços hospitalares e aperfeiçoamento dos materiais de síntese. Todos estes elementos provocaram uma mudança na evolução e no prognóstico destas fraturas, como as menores taxas de necrose avascular que vem sendo relatadas em algumas publicações recentes ${ }^{(16,17)}$.

Com o aumento de nossa casuística, esperamos poder melhor estabelecer alguns aspectos que não ficaram claros na presente análise, como a importância do tratamento precoce e o tipo de tratamento empregado. Desta forma, podemos instituir protocolos terapêuticos visando à minimização das complicações e a melhora dos resultados funcionais em longo prazo. 


\section{CONCLUSÕES}

1 - Entre os 29 pacientes tratados, segundo os critérios de Ratliff, obtivemos 17 (58,6\%) pacientes com bons resultados, oito $(27,6 \%)$ regulares e quatro $(13,8 \%)$ maus resultados.

2 - Quando aplicado o tratamento incruento obtivemos um $(17,0 \%)$ paciente com bom resultado, três (50,0\%) regulares e dois $(33,0 \%)$ maus resultados pela classificação de Ratliff.

3 - Quando aplicado o tratamento cirúrgico obtivemos 16
$(69,3 \%)$ bons resultados, cinco (21,0\%) regulares e dois $(8,7 \%)$ maus resultados pela classificação de Ratliff.

4 - Obtivemos 73,3\% de bons resultados, quando a cirurgia foi realizada nas primeiras 24 horas;

5 - Nos pacientes submetidos à terapêutica operatória, após as primeiras 24 horas, o índice de bons resultados foi de $42,8 \%$.

6 - Pacientes submetidos à cirurgia nas primeiras 24 horas evoluíram com necrose da cabeça do fêmur em $13,3 \%$, enquanto os que foram operados após este período tiveram esta complicação em 21,4\%.

\section{REFERÊNCIAS}

1. Freitas MB, Mothes FC. Fratura do colo do fêmur em crianças. Rev Bras Ortop. 2006;41(5):151-6.

2. Morsy HA. Complications of fracture of the neck of the femur in children. A long-term follow-up study. Injury. 2001;32(1):45-51.

3. Forlin E, Guille JT, Kumar SJ, Rhee KJ. Complications associated with fractures of the neck of the femur in children. J Pediatr Orthop. 1992;12(4):503-9.

4. McDougall A. Fractures of the neck of the femur in childhood. J Bone Joint Surg Br. 1961;43(1):16.

5. Rewers A, Hedegaard H, Lezotte D, Meng K, Battan FK, Emery K, et al. Childhood femur fractures, associated injuries, and sociodemographic risk factors: a population-based study. Pediatrics. 2005;115(5):543-52.

6. Hahn MP, Ostermann PA, Richter D, Dávid A. [Classification, therapy and complications of pediatric femoral neck fractures]. Zentralbl Chir. 1995;120(11):832-40.

7. Mayr J, Hirner V, Styhler W, Posch E, Jelen M, Linhart WE, et al. [Femoral neck fractures in childhood]. Unfallchirurg. 1998;101(6):426-32.

8. Matejka J, Pavelka T, Kostál J, Cervenková H. [Long-term results following fracture of the femoral neck in children]. Acta Chir Orthop Traumatol Cech. 2005;72(2):98-104

9. Colonna PC. Fracture of the neck of the femur in children. Ann Surg. 1929;6:793-7.
10. Ratliff $\mathrm{AH}$. Fractures of the neck of the femur in children. J Bone Joint Surg Br. 1962;44:528-42.

11. Mirdad T. Fractures of the neck of femur in children: an experience at the Aseer Central Hospital, Abha, Saudi Arabia. Injury. 2002;33(9):823-7.

12. Inan U, Köse N, Omeroğlu H. Pediatric femur neck fractures: a retrospective analysis of 39 hips. J Child Orthop. 2009;3(4):259-64.

13. Beaty $\mathrm{JH}$. Fractures of the hip in children. Orthop Clin North Am. 2006;37(2):223-32.

14. Crawford HB. Experience with the non-operative treatment of impacted fractures of the neck of the femur.J Bone joint Surg Am. 1965;47:830-1.

15. Mitchell JI. Fracture of the neck of the femur in children. JAMA. 1936;107:1603-6.

16. Moon ES, Mehlman CT. Risk factors for avascular necrosis after femoral neck fractures in children: 25 Cincinnati cases and meta-analysis of 360 cases. J Orthop Trauma. 2006;20(5):323-9.

17. Flynn JM, Wong KL, Yeh GL, Meyer JS, Davidson RS. Displaced fractures of the hip in children. Management by early operation and immobilization in a hip spica cast. J Bone Joint Surg Br. 2002;84(1):108-12.

18. Boitzy A. Fractures of the proximal femur. In: Weber BG, Brunner C, Freuler F. Treatment of fractures in children and adolescents. New York: Springer Verlag; 1980. p. 254-67.

19. Morsy HA. Complications of fracture of the neck of the femur in children. A long-term follow-up study. Injury. 2001;32(1):45-51. 